\title{
Parotidectomy and neck dissection in locally advanced and relapsed cutaneous squamous cell carcinoma of Head and Neck Region
}

\author{
Giulianno Melo ${ }^{1}$, Luiz Guilherme ${ }^{1}$, Arthur Gatti ${ }^{1,2}$, Juliana Pacheco ${ }^{1}$, Marcel Palumbo ${ }^{1}$, \\ Fabiano Callegari ${ }^{1}$, Marcio Abrahao ${ }^{1}$, and Onivaldo Cervantes ${ }^{1}$ \\ ${ }^{1}$ Federal University of Sao Paulo Paulista Medical School \\ ${ }^{2}$ UNIFESP - Universidade Federal de São Paulo
}

July 27, 2020

\begin{abstract}
Objective: To investigate the prognostic factors to developing parotid and neck metastasis in locally advanced and relapsed cutaneous squamous cell carcinoma (CSCC) of the head and neck region. Study Design: Retrospective cohort study. Setting: Single-center study enrolling consecutive patients with advanced CSCC from 2009 to 2019. Subjects and Methods: Seventy-four cases were identified. Study variables demographic data, clinical skin tumor stage, neck stage, parotid stage (P stage), surgical treatment features, and parotid, regional, and distant metastases. Survival measures: overall survival (OS) and disease-specific survival (DSS). Results: The study group included 72.9\% men (median age, 67 years); 67.5\% showed T2/T3 tumors, 90.5\% comorbidities, $20.2 \%$ immunosuppressed, with median follow-up: 35.8 months. The most frequent skin primary were auricular and eyelid regions, $75 \%$ underwent primary resection with flap reconstruction. Parotid metastasis was present in $50 \%, 32.4 \%$ showing parotid extracapsular spread, multivariate analysis found $\mathrm{OR}=37.6$ of positive parotid metastasis evolve into positive neck metastasis, $\mathrm{p}=0.001$. Occult neck metastasis, neck metastasis, and neck extracapsular spread were observed in $13.5 \%$, $51.3 \%$, and $37.8 \%$, respectively. Kaplan-Meier survival: Clinical T4 and T1, p=0.028, P1 stage: $30 \%$ and $5 \%$ survival at 5 and 10 years, P3 stage: 0\%, $\mathrm{p}=0.016$; OS and DSS showed negative survival for the parotid metastasis group, $\mathrm{p}=0.0283$. Conclusion: Our outcomes support a surgically aggressive approach for locally advanced and relapsed CSCC, with partial parotidectomy for P0, total parotidectomy for P1-3, selective I-III neck dissection for all patients and adjuvant radiochemotherapy to appropriately treat patients with advanced CSCC of the head and neck region.
\end{abstract}

\section{Key words}

Skin Neoplasms

Prognosis

Parotid Neoplasms

Survival analysis

Salivary Gland Diseases

\section{Introduction}

Cutaneous squamous cell carcinoma (CSCC) is the second-most common neoplasia in humans after basal cell carcinoma; about 1.2 million new cases of head and neck skin cancer are estimated in 2040, with 680,000 deaths. ${ }^{1}$ In Brazil, 173,930 new cases of non-melanoma skin cancer are estimated in 2020, with about 2,000 deaths ${ }^{2}$. Approximately $80 \%$ of CSCCs are located in the head and neck region and advanced CSCC have 
a mortality rate of $20 \%$, although this value may have been underestimated due to the lack of precise data in developing countries in South and Central America.

Advanced CSCC is defined as locally invasive in deep anatomic structures with or without presence of regional or distant metastasis; nominated as locally advanced (laCSCC) and metastatic (mCSCC); classified as T3/T4, stage III or IV, where no parotid and locally treatment standards are stablished; the relapsed CSCC (reCSCC) is defined as multiple recurrences after successfully margins free resection ${ }^{6}$. The estimated incidence was 8.000 cases of nodal metastasis and 3.000 deaths annually for both laCSCC and mCSCC whose still are in a standardized area of unmet medical need ${ }^{7}$.

The recommendations of treatment are based on literature review and guidelines, generally, primary surgical excision with safety margins and the appropriate lymph nodes chain dissection are necessary on the proven positive regional metastasis or in the high risk patient, but still, debate exists in the clinically negative neck and the extension of the parotidectomy ${ }^{8}$.

The incidence of parotid metastasis from CSCC is not common, occurring in $1 \%$ to $5 \%$ of all cases of CSCC in the head and neck region and regional metastasis can occur up to five years after resection of the primary ${ }^{3-5}$. The high-risk clinical features for parotid and neck metastasis from CSCC include size $(\mathrm{T})$; depth; scalp, ear pavilion; immunosuppression; recurrence; poor differentiation and others ${ }^{9-12}$. Recently the immunosuppression induced by drug delivery to organ transplants patients, to those with autoimmune diseases and other clinical comorbidities (diabetes mellitus), has been recognized as an important risk factor for the development of CSCC, with growing incidence and mortality in the world ${ }^{13,14}$.

Parotid metastasis from CSCC has an unfavorable prognosis, with lower survival, higher incidence of locoregional recurrence, high extracapsular spread and occult neck metastasis; however, there are few information about the biological tumoral spread from parotid to neck nodes ${ }^{5,11,15-17}$. There are some parotid and neck staging systems, denominating it as positive $(\mathrm{P}+)$ or negative $(\mathrm{P} 0)$; or the N1S3 classification, which does not have consensus worldwide. At present, the most accepted system is the TNM system, although the pN staging evaluation is directly affected by surgical technique, dissection amount, and the quality of pathologic examination $^{18-22}$.

To the present, no consensus exists regarding the parotid treatment and the indication and extent of neck dissection in the reCSCC and laCSCC; the identification of the risk factors for parotid metastasis in reCSCC and la CSCC can allow selecting the proper treatment approach for the parotid gland and to the neck dissection.

\section{Objective}

The objective was to investigate the prognostic factors to developing parotid and neck metastasis in reCSCC and laCSCC of the head and neck region and evaluate the treatment results.

\section{Methods}

This retrospective study with a cohort case-series design was conducted at a single center, public Teaching Hospital with the same assistant surgeons and included referred consecutive patients with laCSCC and reCSCC in the head and neck region with or without parotid or neck metastasis from 2009 to 2019. Institutional ethical review board approval was obtained before beginning this project and the patients provided informed consent. The [removed for blind peer review] Ethics Committee approval number to this study was 0905/2015, CAAE: 48857315.6.0000.5505, in March 2016.

This study was conducted in accordance with the Preferred reporting of case series in surgery (PROCESS) criteria following the PROCESS guidelines ${ }^{23}$; the Guidelines for Cohort studies in Surgery by Agha et al. ${ }^{24}$ and with the Standards for quality improvement reporting excellence guidelines (SQUIRE 2.0) ${ }^{25}$. The study was in accordance with the declaration of Helsinki and has been registered under the WHO Universal Trial Number (UTN) U1111-1249-0072 and the Brazilian Clinical Trials Registry (ReBeC) number RBR-36vxx7. 
Anatomopathological evaluation of tissue samples was according to university standards, all samples of the primary skin tumors and surgical specimens were re-examined by two experienced pathologists and reclassified according to the TNM 8ed. and WHO definition criteria ${ }^{11,20,26,27}$.

After confirmation of CSCC diagnosis, descriptions of patients and tissue samples were recorded, including sex, age, comorbidities, date of diagnosis, mean duration of symptoms, surgical procedure, surgical complications, parotid status, neck lymph node status, clinical (cTNM) and pathological (pTNM) stages as defined by the TNM 8ed. ${ }^{20}$, margin status (positive, negative, exiguous: [?] $5 \mathrm{~mm}{ }^{28}$ ), PNI, ALI, radiotherapy, chemotherapy data, recurrence data, date and patient condition at last consultation. The parotid staging adopted by our service, was done according to the $\mathrm{P}$ stages by O'Brien et al. ${ }^{29}$ : $\mathrm{P} 1$, metastatic $\mathrm{SCC}<3$ $\mathrm{cm}$; P2, tumor 3-6 cm or multiple metastatic parotid nodes; P3: tumor $>6 \mathrm{~cm}$, VII nerve palsy or skull base invasion.

The inclusion criteria were as follows: non-melanoma skin cancer in the head and neck region, recurrence status or advanced primary stage, referred to our multidisciplinary team in the head and neck cancer department, CSCC histologic variants, with or without parotid or neck metastasis, subjected to surgical treatment with curative intent for the skin primary tumor and/or the tumors in the parotid and/or in the neck, with a minimum follow-up of six months.

The exclusion criteria were as follows: Merkell cell carcinoma, melanoma, patients lost to follow-up, incomplete clinical data, surgery performed at other institutions on their primary tumor or neck, distant metastasis at first presentation, refusal to participate in the study, refusal to undergo surgical treatment, and surgery not performed due to advanced clinical status.

Statistical analyses were conducted with the odds ratios to calculate the risk of exposed cases; a twoproportion test to compare the proportions of two variables; the chi-squared test and T-student test with a significance of $\mathrm{p}<0.05$ for comparing frequencies; logistic regression analyses with a significance of $\mathrm{p}<$ 0.05 ; and univariate and multivariate analysis and Kaplan-Meier survival curve analyses.

\section{Results}

Between 2009 and 2019, we identified a total of 91 patients with advanced cutaneous carcinoma, 11 patients with basal cell carcinomas were excluded, 3 were lost to follow-up and 3 others refused surgical treatment. The final group of 74 patients, all with laCSCC or reCSCC in the head and neck region, was designated as the study group and divided according to parotid metastases status into positive parotid metastasis group (PM) with 37 patients who was compared to the without parotid metastases (WPM) group with 37 patients.

\section{Patients and Clinical Characteristics}

The demographic, clinical, and surgical characteristics of the two groups are shown in Table 1 .

The study group included $72.9 \%$ men with a median age of 67 years (range, 23 to 103 years). The distribution of primary clinical skin $\mathrm{T}$ stages was in Table 1, T2 and T3 were more prevalent. The locations of these were: auricular, $22.9 \%$; scalp, $14.8 \%$; frontal, 10.8\%; eyelid, $22.9 \%$; malar, $13.5 \%$; and nose, $9.4 \%$.

Comorbidities were observed in $90.5 \%$ of the patients; $20.2 \%$ immunosuppressed due to some kind of transplant. The mean follow-up was 35.8 months.

\section{Treatment}

The primary advanced skin cancer surgery was total resection with flap reconstruction: $48.6 \%$; exenteration: 13.5\%; temporalectomy: 13.5\%; rhinectomy: $5.4 \%$ (Table 1). Histological differentiation was classified as follows: well, $35.1 \%$; moderate, $43.2 \%$; and poor, $21.6 \%$. The surgical margins were compromised in $29.7 \%$ (Table 2) .

The overall incidence of neck metastasis was $32.4 \%$ : 51.4\% in PM group and 13.5\% in WPM group. Of these, $22.9 \%$ showed positive neck extracapsular spread. The type of neck dissection was selective (45.9\%), modified radical (37.8\%), or classic radical (8.1\%) (Tables 1 and 2). 
The overall incidence of parotid metastasis was $50.0 \%$; partial parotidectomy was done as follows: PM: 43.2\% and WPM: 29.7\%, total parotidectomy: PM: $54.1 \%$ and WPM: $10.8 \%$. The parotid P stage was in PM group: P1 (54.1\%), P2 (43.2\%), P3 (2.7\%); in WPM group: P1: 0\%, P2 (2.7\%), P3 (0\%) (Table 2) . Overall parotid metastasis extracapsular spread was present in $32.4 \%$, the PM group showed total parotidectomy: $54.1 \%$, positive ECE: $35.1 \%$, compromised margins: $29.7 \%$; and partial parotidectomy: 43,2\%, positive ECE: $27.0 \%$, compromised margins: $24.3 \%$.

The N stage was N0 (60.8\%), N1 (18.9\%), N2 (20.2\%). Of these, $22.9 \%$ of the patients presented with lymph node extracapsular spread.

Adjuvant treatment was administered to $72.9 \% ; 62.1 \%$ underwent external radiotherapy alone, none have received braquitherapy; while $18.9 \%$ received chemoradiotherapy (Table 1) .

\section{Outcomes}

The overall recurrence rate (local and regional) was $22.9 \%$ and $10.8 \%$ for distant metastasis (Table 1). In the PM group, $51.4 \%$ of the patients also showed neck metastasis. The rate of neck occult nodal disease was $13.5 \%(5 / 37)$. Mortality rate related to the disease was $32.4 \%$; the morbidity rate (alive with neoplasia) was $27.0 \%$; and only $40.5 \%$ of the patients were alive without disease, thus, the survival rate was $67.6 \%$.

The Table 3 shows the univariate analysis, demonstrating differences in relation to extracapsular spread from neck, extracapsular spread from parotid, and compromised margins. In Table 4, Logistic regression multivariate analysis showed that parotid metastasis extracapsular spread was significantly associated with evolution to positive neck metastasis $(\mathbf{O R}=\mathbf{3 7 . 6 1})$.

The survival curves showed no differences in comparisons based on the type of parotid surgery; histological differentiation degree; recurrence of primary CSCC in a different location; or pathological final stage (I/II x III/IV) in both groups.

\section{Disease Survival}

The specific survival curve for clinical T stage (Figure 1 ) showed significant differences in relation to clinical T stage ( $\mathrm{T} 4$ vs. T1 $\mathbf{p}=\mathbf{0 . 0 2 8}$ ) .

The Kaplan-Meier OS curve showed a significantly worse prognosis (log-rank; $\mathbf{p}=\mathbf{0 . 0 2 8 3}$ ) in the PM group (Figure 2). The OS rates at 3 years, 5 years, and 10 years in the PM group were $58 \%, 46 \%$, and $13 \%$, respectively, while those in the WPM group were $78 \%, 72 \%$, and $72 \%$, respectively.

Kaplan-Meier analysis of DSS on the P stage in the PM group showed significant differences: P1 and P2 at 3,5 , and 10 years were respectively $40 \%, 30 \%$, and $5 \%$; and $6 \%, 0 \%$, and $0 \%$. The values for P3 were $0 \%$ at all corresponding periods of time $(\mathbf{p}=\mathbf{0 . 0 1 6} ;$ Figure 3$)$.

\section{Discussion}

\section{Synopsis of new findings}

Our cohort study assessed 74 patients with laCSCC or reCSCC of head and neck region, with a median follow-up of 35.8 months; $50 \%$ showed parotid and/or neck metastasis, much higher than the $20 \%$-39\% range of literature, explained by health system failures and the continental size of country located in tropical area $18,30,31$. The majority of the patients were men, median age of $67 \mathrm{y}$, explained by prolonged sun occupational exposure.

In our cases, the prevalent location of the primary CSCC was the auricular and eyelid region, almost $67.5 \%$ were $\mathrm{T} 2$ and $\mathrm{T} 3$, some $\mathrm{T} 1$ reCSCC evolved with parotid metastasis, probably due immunosuppression condition, contrary to some authors (Table $\mathbf{1})^{30,32}$. In univariate and multivariate analysis, primary tumor site, histopathological characteristics and margins did not affect the OS, attributed to retrospective data bias $^{33-35}$. Interestingly, our study found $90.5 \%$ of patients with comorbidities (20\% immunosuppressed due 
transplant organ), having main role on developing progressively metastases from primary to parotid/neck 36-38.

In $75 \%$ of patients, the main surgery was total primary resection with reconstruction followed by exenteration and temporalectomies, showing an aggressive presentation, similar to other developing $\operatorname{countries}^{35}$. Our data did not find an association between the type of primary surgery and survival, unusual since such a correlation is expected, however, there is comparable studies ${ }^{34,38,39}$.

The margins was compromised in $43.3 \%$ in the PM group and only $16.2 \%$ in the WPM group, expected in advanced cases, with no impact on survival. Majority of PM group patients had clinical T3/T4 (86.4\%) tumors (most laCSCC) instead the WPM group (most reCSCC) showed T1/T2 (86.4\%); this biological behavior difference has impacted on survival (T1 x T4) curves, with clearly negative impact on the clinical T stage survival, $\mathrm{p}=0.028$ (Figure 1) .

The parotid metastasis from CSCC has an unfavorable prognosis $17,18,21,42$. Our study reported a $50 \%$ incidence of parotid metastasis in laCSCC and reCSCC, 32.4\% showed parotid extracapsular spread, with $29.7 \%$ compromised margins, denoting a difficulty to achieve an adequate surgical treatment ${ }^{10,14,21,36,43-46}$; otherwise, our isolated occult parotid metastasis rate was only $2.7 \%$, meaning that probably is safe to observe the clinically and radiologically negative parotid gland lymph node cases (Table 2 and Table3 ).

This is very controversial, although no difference was observed between types of parotid surgery, our overall recurrence rate was $22.9 \%$, the most frequent in parotid surgical bed $(21.6 \%)$.

Our overall rate of occult neck metastasis was 13.5\%; in PM group, 51.3\% presented clinical neck metastasis, and $37.8 \%$ of these, extracapsular spread; demonstrating the path of tumor behavior; once it has the positive parotid gland metastasis, rapidly evolves to a positive neck metastasis; in fact, $64.8 \%$ of PM group has the N1-N2 neck compared to the 13.5\% N1-N2 necks in the WPM group (Table 2 ); notably, the risk of a positive parotid metastasis evolving into positive neck metastasis was 37.6, with p=0.001 (Table 4 ).

Our OS curve (Figure 2 ) demonstrated negative survival for the PM group, with worst predictors $(\mathrm{p}=0.0283)$. Once the parotid shows clinical positive metastasis ( $\mathrm{P} 1-3$ stages) the disease-specific survival is progressively and severely impacted (Figure 3) ( $\mathrm{p}=0.016)$, findings similar to others $18,31,32,36,40,42,43,45-48$.

Although $72.9 \%$ of our patients underwent to adjuvant radiochemotherapy, there have not impacted on survival, maybe due the poor response of CSCC to chemoradiation (Table 4) $10,32,35,36,46$, but the radiochemotherapy is still the adjuvant treatment in multiple guidelines $12,21,31,32,36,49$.

The Table 5 show the review of literature in 20 years regarding overall survival, and parotid and neck metastasis in CSCC, including our data.

\section{Clinical Applicability}

The high levels of recurrence have guided us to adopt aggressive initial approach: Partial Parotidectomy to P0 patients and Total Parotidectomy to P1-3 patients; once the survival was poor in the P1-P3 patients and there is not a difference in recurrence and survival between types of parotidectomy; at same time we lowering the facial nerve complications in P0 stages.

We also suggest the selective (I-III) neck dissection in all cases in order to achieve prolonged survival since it is difficult to "wait and see" the neck.

\section{Conclusion}

Our study demonstrated, in laCSCC and reCSCC, 50\% of patients with parotid metastasis with $32.4 \%$ extracapsular parotid metastasis spread; $13.5 \%$ overall rate of neck occult metastasis, $51.3 \%$ clinical neck metastasis with $37.8 \%$ neck extracapsular spread. The risk of positive parotid metastasis evolving into positive neck metastasis was 37.6. The clinical skin T4 tumor and presence of parotid metastasis negatively impacted patient survival; P1 stage resulted in $30 \%$ and $5 \%$ survival at 5 years and 10 years, respectively. Our 
outcomes support the surgically aggressive approach for laCSCC and reCSCC, with partial parotidectomy for P0 and total parotidectomy for P1-3 stages and selective neck dissection I-III in all patients.

\section{Disclosures}

Competing interests: None.

Sponsorships: None.

Funding source: None.

\section{References}

1. Bray F, Ferlay J, Soerjomataram I, Siegel RL, Torre LA, Jemal A. Global cancer statistics 2018: GLOBOCAN estimates of incidence and mortality worldwide for 36 cancers in 185 countries. CA Cancer J Clin 2018;68:394-424.

2. Instituto Nacional de Cancer. Estimativa 2020: incidencia de cancer no Brasil. Rio de Janeiro INCA; 2020 .

3. Rowe DE, Carroll RJ, Day CL Jr. Prognostic factors for local recurrence, metastasis, and survival rates in squamous cell carcinoma of the skin, ear, and lip. Implications for treatment modality selection. $J \mathrm{Am}$ Acad Dermatol. 1992;26(6):976-990.

4. Karia SP, Han J, Schmults CD. Cutaneous Squamous cell carcinoma: estimated incidence of disease, nodal metastasis, and deaths from disease in the united states. JAMA Dermatol. 2013;3:957-966.

5. Goh RY, Bova R, Fogarty GB. Cutaneous squamous cell carcinoma metastatic to parotid - analysis of prognostic factors and treatment outcome. World J Surg Oncol 2012;25(10):117.

6. Stratigos AJ, Garbe C, Dessinioti C, et al. European interdisciplinary guideline on invasive squamous cell carcinoma of the skin: Part 1. epidemiology, diagnostics and prevention. Eur J Cancer 2020;26(20):3001830016 .

7. Jennings L, Schmults CD. Management of High-Risk Cutaneous Squamous Cell Carcinoma. J Clin Aesthet Dermatol. 2010;3(4):39-48.

8. Stratigos AJ, Garbe C, Dessinioti C, et al. European interdisciplinary guideline on invasive squamous cell carcinoma of the skin: Part 2. Treatment. Eur J Cancer 2020;26(20):30019-30018.

9. Cassarino DS, Derienzo DP, Barr RJ. Cutaneous squamous cell carcinoma: a comprehensive clinicopathologic classification. Part one.J CutanPathol. 2006;33:191-206.

10. Swenny L, Zimmerman T, Carroll WR, Schmalbach CE, Day KE, Rosenthal EL. Head and neck cutaneous squamous cell carcinoma requiring parotidectomy: prognostic indicators and treatment selection.Otolaryngol Head Neck Surg. 2014;150:610-617.

11. Vauterin TJ, Veness MJ, Morgan GJ, Poulsen MG, O'Brien CJ. Patterns of lymph node spread of cutaneous squamous cell carcinoma of the head and neck. Head Neck. 2006;28(9):785-791.

12. Bobin C, Ingrand P, Dréno B, Rio E, Malard O, Espitalier F. Prognostic factors for parotid metastasis of cutaneous squamous cell carcinoma of the head and neck. European Annals of Otorhinolaryngology, Head and Neck Diseases,. 2018;135(2):99-103.

13. Gurney B, Newlands C. Management of regional metastatic disease in head and neck cutaneous malignancy. 1. Cutaneous squamous cell carcinoma. Br J Oral Maxillofac Surg. 2014;52:294-300.

14. Veness MJ, Palme CE, Morgan GJ. High-risk cutaneous squamous cell carcinoma of the head and neck: results from 266 treated patients with metastatic lymph node disease. Cancer 2006;106(11):2389-2396. 
15. Lee S, Kim GE, Park CS, et al. Primary squamous cell carcinoma of the parotid gland. Am J Otolaryngol. 2001;22:400-406.

16. Ch'ng S, Maitra A, Allison RS, et al. Parotid and cervical nodal status predict prognosis for patients with head and neck metastatic cutaneous squamous cell carcinoma. J Surg Oncol2008;98(2):101-105.

17. Brantsch KD, Meisner C, Schönfisch B, et al. Analysis of risk factors determining prognosis of cutaneous squamous-cell carcinoma: a prospec-tive study. Lancet Oncol. 2008;9:713-720.

18. O'Brien CJ, McNeil EB, McMahon JD, Pathak I, Lauer CS. Incidence of cervical node involvement in metastatic cutaneous malignancy involving the parotid gland. Head Neck. 2001;23:744-748.

19. Forest VI, Clark JJ, Veness MJ, Milross C. N1S3: a revised staging system for head and neck cutaneous squamous cell carcinoma with lymph node metastases: results of 2 Australian Cancer Centers. Cancer.2010;116(5):1298-1304.

20. Amin MB, Edge S, Greene F, et al. AJCC Cancer Staging Manual (8th edition). Springer International Publishing: American Joint Commission on Cancer; 2017; 2017.

21. Thom JJ, Moore EJ, Price DL, Kasperbauer JL, Starkman SJ, Olsen KD. The Role of Total Parotidectomy for Metastatic Cutaneous Squamous Cell Carcinoma and Malignant Melanoma. JAMA Otolaryngol Head Neck Surg. 2014;140(6):548-554.

22. Wei-Chin Chang, Chun-Shu Lin, Cheng-Yu Yang, Chih-Kung Lin, Yuan-Wu Chen. Lymph node density as a prognostic predictor in patients with betel nut-related oral squamous cell carcinoma. Clin Oral Invest.2018;22:1513-1521.

23. Agha RA, Borrelli MR, Farwana R, et al. Statement: Updating Consensus Preferred Reporting Of CasE Series in Surgery (PROCESS) Guidelines. International Journal of Surgery. 2018;60:279-282.

24. Agha RA, Borrelli MR, Vella-Baldacchino M, Thavayogan R, Orgill DP, STROCSS Group. The STROCSS statement: Strengthening the Reporting of Cohort Studies in Surgery. Int J Surg 2017;46:198-202.

25. Ogrinc G, Davies L, Goodman D, Batalden P, Davidoff F, Stevens D. SQUIRE 2.0 (Standards for QUality Improvement Reporting Excellence): revised publication guidelines from a detailed consensus process. $B M J$ Quality \& Safety. 2016;25:986-992.

26. National Comprehensive Cancer Network (NCCN). National Comprehensive Cancer Network (NCCN). NCCN Clinical Practice Guidelines in Oncology. Squamous Cell Skin Cancer. Version 1.2020. National Comprehensive Cancer Network. https://www.nccn.org/professionals/physician_gls/pdf/squamous.pdf. Published 2019. Accessed2020.

27. Keohane SG, Proby CM, Newlands C, Motley RJ, Nasr I, Mohd Mustapa MF. The new 8th edition of TNM staging and its implications for skin cancer: a review by the British Association of Dermatologists and the Royal College of Pathologists, U.K. Br J Dermatol.2018;179(4):824-828.

28. Batsakis JG. Surgical excision margins: a pathologist's perspective.Advances in Anatomic Pathology. 1999;6(3):140-148.

29. O'Brien CJ, McNeil EB, McMahon JD, Pathak I, Lauer CS, Jackson MA. Significance of clinical stage, extent of surgery, and pathologic findings in metastatic cutaneous squamous carcinoma of the parotid gland. . Head Neck. 2012;24(5):417-422.

30. Myers LL, Ahn C. Cutaneous squamous cell carcinoma metastasis to the parotid region lymph nodes. Laryngoscope 2019;129(7):1579-1586.

31. Košec A, Svetina L, Lukšić I. Significance of clinical stage, extent of surgery and outcome in cutaneous squamous cell carcinoma of the head and neck. International Journal of Oral and Maxillofacial Surgery. $2013 ; 42(1): 82-88$. 
32. Newlands C, Currie R, Memon A, Whitaker S, Woolford T. Non-melanoma skin cancer: United Kingdom National Multidisciplinary Guidelines.J Laryngol Otol 2016;130(S125-S132).

33. Creighton F, Lin A, Leavitt E, Lin D, Deschler D, Emerick K. Factors affecting survival and locoregional control in head and neck cSCCA with nodal metastasis. Laryngoscope 2018;128(8):1881-1886.

34. Bachar G, Mizrachi A, Rabinovics N, et al. Prognostic factors in metastatic cutaneous squamous cell carcinoma of the head and neck.Ear Nose Throat J. 2016;95(10-11):E32-E36.

35. Coombs AC, Butler A, Allison R. Metastatic cutaneous squamous cell carcinoma of the parotid gland: prognostic factors. J Laryngol Otol 2018;132(3):264-269.

36. Skulsky SL, O'Sullivan B, McArdle O, et al. Review of high-risk features of cutaneous squamous cell carcinoma and discrepancies between the American Joint Committee on Cancer and NCCN Clinical Practice Guidelines In Oncology. Head Neck 2017;39(3):578-594.

37. Varra V, Woody NM, Reddy C, et al. Suboptimal Outcomes in Cutaneous Squamous Cell Cancer of the Head and Neck with Nodal Metastases.Anticancer Res 2018;38(10):5825-5830.

38. Sullivan CB, Andresen NS, Kendell N, Al-Qurayshi Z, Pagedar NA. Survival Outcomes for Advanced Cutaneous Squamous Cell Carcinoma of the Head and Neck. Ann Otol Rhinol Laryngol. 2019;128(10):949955.

39. Sweeny L, Zimmerman T, Carroll WR, Schmalbach CE, Day KE, Rosenthal EL. Head and neck cutaneous squamous cell carcinoma requiring parotidectomy: prognostic indicators and treatment selection. Otolaryngol Head Neck Surg. 2014;150(4):610-617.

40. Makki FM, Mendez AI, Taylor SM, et al. Prognostic factors for metastatic cutaneous squamous cell carcinoma of the parotid. J Otolaryngol Head Neck Surg. 2013;5(42):14-22.

41. Iyer NG, Clark JR, Murali R, Gao K, O'Brien CJ. Outcomes following parotidectomy for metastatic squamous cell carcinoma with microscopic residual disease: implications for facial nerve preservation. Head Neck 2009;31(1):21-27.

42. O'Brien CJ, McNeil EB, McMahon JD, Pathak I, Lauer CS, Jackson MA. Significance of clinical stage, extent of surgery, and pathologic findings in metastatic cutaneous squamous carcinoma of the parotid gland. Head Neck 2002;24(5):417-422.

43. D'Souza J, Clark J. Management of the neck in metastatic cutaneous squamous cell carcinoma of the head and neck. Curr Opin Otolaryngol Head Neck Surg 2011;19(2):99-105.

44. Creighton F, Lin A, Leavitt E, Lin D, Deschler D, Emerick K. Factors affecting survival and locoregional control in head and neck cSCCA with nodal metastasis. Laryngoscope. 2018;128(8):1881-1886.

45. Audet N, Palme CE, Gullane PJ, et al. Cutaneous metastatic squamous cell carcinoma to the parotid gland: analysis and outcome. Head Neck. 2004;26(8):727-732.

46. Andruchow JL, Veness MJ, Morgan GJ, et al. Implications for clinical staging of metastatic cutaneous squamous carcinoma of the head and neck based on a multicenter study of treatment outcomes. Cancer.2006;106(5):1078-1083.

47. Speight PM, Barrett AW. Salivary gland tumor. Oral dis.2002;9:229-240.

48. Palme CE, O’Brien CJ, Venesse CJ, McNeil EB, Bron LP, GJ M. Extent of Parotid Disease Influences Outcome in Patients With Metastatic Cutaneous Squamous Cell Carcinoma. Arch Otolaryngol Head Neck Surg. 2003;129(7):750-753.

49. Skulsky SL, O'Sullivan B, McArdle O, et al. Review of high-risk features of cutaneous squamous cell carcinoma and discrepancies between the American Joint Committee on Cancer and NCCN Clinical Practice Guidelines In Oncology. Head Neck. 2017;39(3):578-594. 

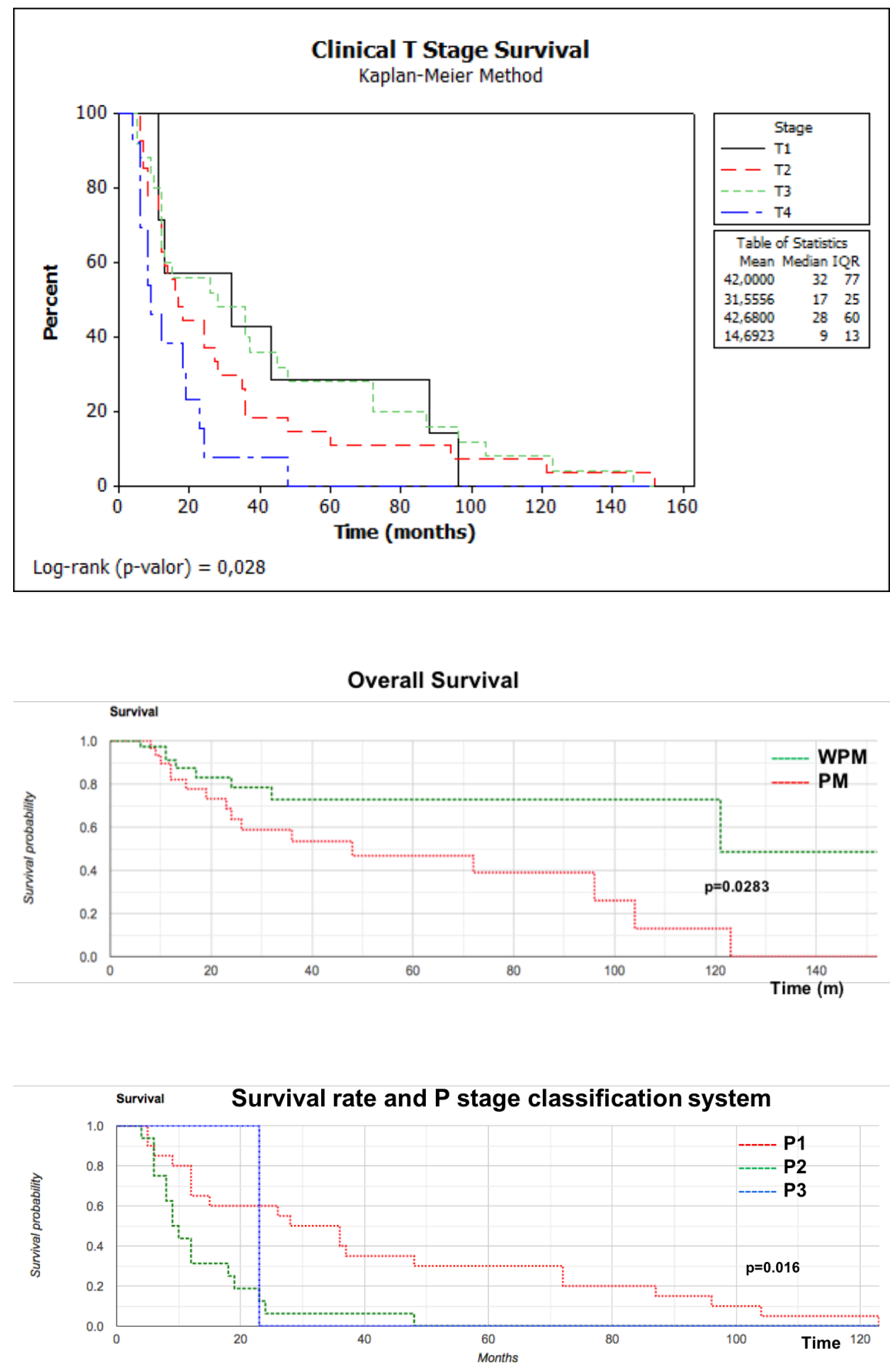

\section{Hosted file}

Tables and Figures Legends-July-20.docx available at https://authorea.com/users/346290/ articles/472405-parotidectomy-and-neck-dissection-in-locally-advanced-and-relapsedcutaneous-squamous-cell-carcinoma-of-head-and-neck-region 